\title{
Lung clearance index is a sensitive, repeatable and practical measure of airways disease in adults with cystic fibrosis
}

\author{
A R Horsley, ${ }^{1,5}$ P M Gustafsson, ${ }^{2}$ K A Macleod, ${ }^{3,5}$ C Saunders, ${ }^{4,5}$ A P Greening, ${ }^{1,5}$ \\ D J Porteous, ${ }^{1,5} \mathrm{~J}$ C Davies, ${ }^{4,5}$ S Cunningham, ${ }^{3,5}$ E W F W Alton, ${ }^{4,5} \mathrm{~J} \mathrm{~A} \mathrm{Innes^{1,5 }}$
}

\begin{abstract}
See related editorial page 98
- Additional information is published online only at http:// thorax.bmj.com/content/vol63/ issue2

${ }^{1}$ School of Clinical and Molecular Medicine, University of Edinburgh, Edinburgh, UK;

${ }^{2}$ Queen Silvia Children's

Hospital, Gothenburg, Sweden;

${ }^{3}$ Royal Hospital for Sick

Children, Edinburgh, UK;

${ }^{4}$ Department of Gene Therapy,

National Heart and Lung

Institute, Imperial College,

London, UK; ${ }^{5}$ UK Cystic Fibrosis

Gene Therapy Consortium, UK
\end{abstract}

Correspondence to:

Dr A R Horsley, Medical

Genetics, Molecular Medicine

Centre, University of Edinburgh,

Western General Hospital,

Edinburgh EH4 2XU, UK; alex.

horsley@ed.ac.uk

Received 10 May 2007

Accepted 19 July 2007

Published Online First

3 August 2007

\section{ABSTRACT}

Background: Lung clearance index $(\mathrm{LCl})$ is a sensitive marker of early lung disease in children but has not been assessed in adults. Measurement is hindered by the complexity of the equipment required. The aims of this study were to assess performance of a novel gas analyser (Innocor) and to use it as a clinical tool for the measurement of $\mathrm{LCl}$ in cystic fibrosis (CF).

Methods: LCl was measured in 48 healthy adults, 12 healthy school-age children and 33 adults with CF by performing an inert gas washout from $0.2 \%$ sulfur hexafluoride $\left(\mathrm{SF}_{6}\right) . \mathrm{SF}_{6}$ signal:noise ratio and $10-90 \%$ rise time of Innocor were compared with a mass spectrometer used in similar studies in children.

Results: Compared with the mass spectrometer, Innocor had a superior signal:noise ratio but a slower rise time (150 ms vs $60 \mathrm{~ms}$ ) which may limit its use in very young children. Mean (SD) LCl in healthy adults was significantly different from that in patients with CF: 6.7 (0.4) vs 13.1 (3.8), $p<0.001$. Ten of the patients with CF had forced expiratory volume in $1 \mathrm{~s} \geqslant 80 \%$ predicted but only one had a normal $\mathrm{LCl}$. $\mathrm{LCl}$ repeats were reproducible in all three groups of subjects (mean intra-visit coefficient of variation ranged from $3.6 \%$ to $5.4 \%$ ).

Conclusions: Innocor can be adapted to measure $\mathrm{LCl}$ and affords a simpler alternative to a mass spectrometer. $\mathrm{LCl}$ is raised in adults with CF with normal spirometry, and may prove to be a more sensitive marker of the effects of treatment in this group.

As part of a programme aimed at measuring the response to gene therapy in cystic fibrosis (CF), we are interested in developing more sensitive measures of changes in CF airway function and structure. In the USA the only marker of lung function currently recognised as a primary end point in CF trials is the forced expiratory volume in $1 \mathrm{~s}\left(\mathrm{FEV}_{1}\right){ }^{1}$ In early disease this reflects total airways resistance and is insensitive to changes in small airways, which contribute $<10 \%$ of the overall resistance in healthy adult subjects. ${ }^{2}$ Significant structural airway damage can be demonstrated on CT scanning in the presence of a normal $\mathrm{FEV}_{1}{ }^{3}$

In early lung disease, ventilation heterogeneity results from regional differences in small airway calibre (those beyond division 8). ${ }^{45}$ This can be demonstrated both in computer models of the human lung ${ }^{6}$ and from in vivo $M R$ images $^{7}$ or radiolabelled tracer gas distribution. ${ }^{78}$ Inert gas washout is an alternative technique which involves measuring the elimination of a non-absorbed gas as it is exhaled during tidal breathing. The gas can either be resident nitrogen washed out by breathing $100 \%$ oxygen, or an exogenous tracer gas which has first been breathed in to equilibrium. Lung clearance index (LCI), a simple marker of deranged ventilation, can be calculated from the washout curves. ${ }^{9}$ Past studies using a variety of methods have shown that LCI is reproducible and more sensitive than $\mathrm{FEV}_{1}$ at identifying early lung disease in children. ${ }^{9-13}$ In addition, Aurora et a ${ }^{10}$ showed that LCI is further raised in children infected with Pseudomonas aeruginosa and Kraemer et $a l^{13}$ showed LCI to be an early predictor of deteriorating lung function in children.

Although an old technique, ${ }^{14}$ measurement of LCI has always relied upon complex and bulky equipment, usually assembled from separate components by the investigators themselves, and has largely been restricted to a research setting. The current best method involves using a mass spectrometer (MS) to measure the washout of the inert tracer gas sulphur hexafluoride $\left(\mathrm{SF}_{6}\right)$. Although these are simpler than nitrogen washouts, MS are expensive to purchase and maintain.

The purpose of this study was to investigate LCI in healthy subjects and adults with CF using a modified Innocor device (Innovision, Odense, Denmark). Innocor is a compact gas analyser and flow sensor originally designed to measure cardiac output by inert gas rebreathing. The gas analyser uses photoacoustic spectroscopy to measure several gases including low concentrations of the inert tracer $\mathrm{SF}_{6}$, making it a suitable device for ventilation distribution measurements. More information on the gas analyser is given in the online supplement (pages 2-5).

In preparation for its use as an end point in clinical trials, the aims of this study were:

1. To compare the performance (assessed by response time and signal:noise ratio) of the Innocor gas analyser with that of the current inert gas washout standard (MS).

2. To adapt the Innocor device and analysis software into a clinical system for measurement of functional residual capacity (FRC) and LCI.

3. To assess how LCI changes with age of subject in healthy volunteers.

4. To assess the intra and inter-visit reproducibility of LCI in healthy volunteers.

5. To use the adapted Innocor to measure FRC and LCI in normal adult subjects and patients with CF and to compare LCI with spirometry. 


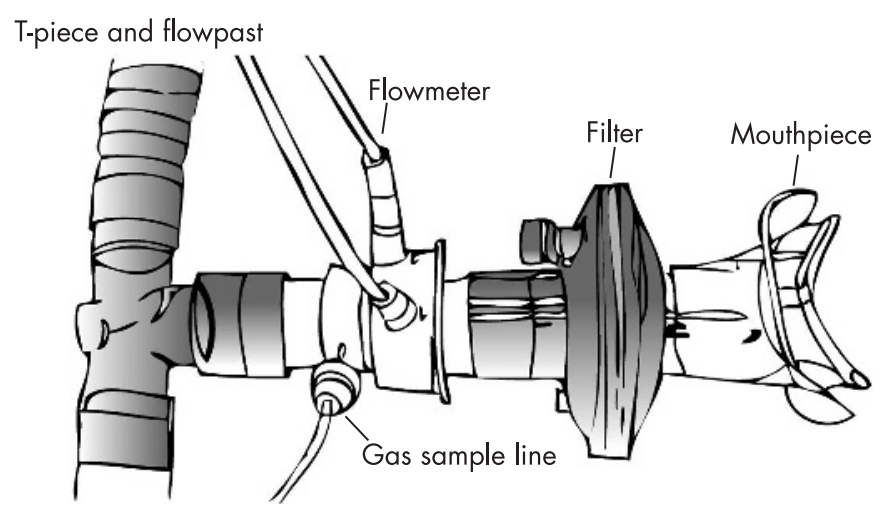

Figure 1 Patient interface used for inert gas washout with Innocor gas analyser.

\section{METHODS}

\section{Equipment}

To measure LCI, a mouthpiece fitted with a flowmeter and gas sampling port is required (fig 1). This is connected to a detachable flowpast tube which is used to supply tracer gas during the wash-in and is then removed at the start of washout. A more detailed Methods section is available in the online supplement, and the modifications to the standard Innocor patient interface are described in detail on pages 5-7.

Spirometry was measured according to American Thoracic Society/European Respiratory Society guidelines; ${ }^{15}$ predicted values for $\mathrm{FEV}_{1}$ are those provided by the European Community for Coal and Steel (adults $\geqslant 17$ years) ${ }^{16}$ and Rosenthal et al (children $\leqslant 16$ years). ${ }^{17}$

\section{Performance of Innocor gas analyser}

The signal:noise ratio at the start and end of a washout and the rise time of the gas analyser in response to a step change in $\mathrm{SF}_{6}$ concentration were assessed as described in the online supplement (page 8). Performance was compared with that of a MS used routinely for LCI measurements. The ability of the complete modified system to integrate flow and gas signals accurately was assessed using a gas calibration syringe which can be set to deliver different volumes (Hans Rudolph, Missouri, USA). This was filled with $0.2 \% \mathrm{SF}_{6}$ in air (BOC, Guildford, UK) to a range of different starting volumes and a washout performed by incomplete filling and emptying of the syringe around this starting point. The syringe volume derived from the calculated "expired" volume of $\mathrm{SF}_{6}$ was then compared with the known starting volume.

Flow and $\mathrm{SF}_{6}$ data were exported for analysis on custom-built software. FRC was derived from the total expired $\mathrm{SF}_{6}$ volume, calculated by integration of flow and $\mathrm{SF}_{6}$ signals. $\mathrm{LCI}$ is defined as the number of lung turnovers (ie, multiples of FRC) required to reduce end tidal marker gas concentration to $1 / 40$ th of the starting value (as described in the online supplement, page 11).

\section{Subjects}

Forty-nine healthy non-smokers ( $<10$ pack-years smoking history) with no active lung disease and on no regular respiratory medications were recruited as normal adult volunteers (age range 19-58 years). Thirteen healthy child volunteers (age range 6-16 years) were recruited if they had no previous diagnosis of recurrent wheeze or asthma and were taking no current inhaled medication. There was no history of significant respiratory disease requiring hospitalisation (eg, pneumonia, pertussis, tuberculosis), no prematurity ( $<34$ weeks gestation) and no significant co-morbidity. Thirty-three patients with CF (age range 17-49 years) were recruited from the Scottish Adult CF Service, the diagnosis being based on a combination of clinical presentation and sweat testing and confirmed by genotyping. All volunteers, patients and (where relevant) parents provided informed consent. Paediatric volunteers provided assent where appropriate. This study was approved by the Lothian research and ethics committee.

\section{Washout test}

Subjects were seated and suitably distracted by watching television. A noseclip was applied and tidal breathing established while the subject was connected to the flowpast circuit containing $0.2 \% \mathrm{SF}_{6}$ in air. This was supplied from a compressed gas cylinder with the flow rate adjusted to ensure that rebreathing did not occur. This wash-in phase continued for at least $5 \mathrm{~min}$ in adults or $4 \mathrm{~min}$ in children under 16 years and, in all cases, until inspiratory and expiratory $\mathrm{SF}_{6}$ concentrations differed by $<0.004 \%$ (absolute difference in $\mathrm{SF}_{6}$ concentration). The flowpast circuit was then detached during expiration and the washout measured until the end tidal $\mathrm{SF}_{6}$ had fallen to less than $1 / 40$ th of the starting concentration (ie, $<0.005 \%$ ). In healthy children ( $<16$ years) an identical gas analyser and protocol were employed at a separate research site, but a smaller filter was used to reduce the precapillary dead space $(36 \mathrm{ml}$ vs $46 \mathrm{ml}$ in adults).

Subjects completed three sets of wash-ins and washouts. A washout was discarded if the resulting calculated FRC differed by $>10 \%$ from both the other two repeats. ${ }^{18}$

\section{Statistical analysis}

Data were analysed using Prism (GraphPad Software Inc, California, USA). The results are given as mean (SD) unless otherwise stated. Within-test repeatability for LCI was determined by calculating the coefficient of variation (CV) as $100 \times$ $\mathrm{SD} /$ mean. Inter-visit reproducibility was assessed using the Bland-Altman technique. Correlation with age and height were assessed by multiple linear regression analysis. Numerical values for LCI and $\mathrm{FEV}_{1} \%$ predicted were compared using a MannWhitney $U$ test. The $95 \%$ limits of normality for LCI were calculated as mean $\pm 1.96 \times$ residual standard deviations. A $p$ value of $<0.05$ was considered statistically significant.

\section{RESULTS}

\section{Technical validation of Innocor device}

Signal:noise ratio of Innocor and MS

The Innocor device has a lower gas concentration operating range than the MS. Signal quality is therefore given at the starting and finishing concentrations of a washout, which are different for the two devices (table 1). For both devices there is a fall in signal:noise ratio as the gas concentration falls, but the Innocor signal quality remains superior throughout, despite much lower $\mathrm{SF}_{6}$ concentrations.

\section{Rise time and delay of gas signal}

The mean (SD) $\mathrm{SF}_{6} \quad 10-90 \%$ rise time was 154 (5) ms for Innocor and $64(5) \mathrm{ms}$ for the MS ( $\mathrm{p}<0.001)$. The longer rise time of the Innocor gas analyser was allowed for by offsetting gas and flow signals during analysis by an additional $50 \mathrm{~ms}$. This corresponds to the $50-80 \%$ rise time of the gas signal, and has the effect of speeding the response time by realigning the flow signal with the $80 \%$ response fraction of the gas signal. ${ }^{19}$ 
Table 1 Signal:noise ratios of Innocor and mass spectrometer (MS) at gas concentrations encountered at start and end of washout

\begin{tabular}{llllll}
\hline & \multicolumn{2}{l}{$\mathbf{S F}_{\mathbf{6}}$ concentration $(\%)$} & & \multicolumn{2}{l}{ Signal:noise ratio } \\
\cline { 2 - 3 } & $\mathbf{S t a r t}$ & Start & End \\
\hline MS & 4.0 & 0.1 & 200 & 13 \\
Innocor & 0.2 & 0.005 & 944 & 53 \\
\hline
\end{tabular}

The signal:noise ratio is calculated as the ratio of mean to standard deviation of a stable gas signal over $10 \mathrm{~s}$.

The accuracy of this adjustment was then confirmed by integration of known volumes of $\mathrm{SF}_{6}$ from a calibration syringe.

\section{Validation of FRC measurements}

Sixteen washouts were performed using a calibration syringe with the starting volume varied between 1.5 and 3 litres. There was good agreement between the measured and actual syringe volumes (see fig 1 in online supplement II). The mean (SE) error between measured and actual syringe volume was $16.3(2.4) \mathrm{ml}$ or $1.1(0.2) \%$.

\section{In vivo $\mathbf{L C I}$ measurement}

LCI was assessed successfully in 12 healthy children, 48 adult healthy volunteers and 33 adults with CF. The demographic data of the study subjects are given in table 2. Data from two additional healthy volunteers (one adult, one child) could not be analysed because of technical difficulties (see below).

\section{Effect of age, height and gender on $\mathrm{LCl}$ in non-CF subjects}

Figure 2 shows the relationship between LCI and age (min 6 years, max 58 years). In those aged $>16$ years there was no relationship between LCI and age. When the two cohorts were combined there was a weak but statistically significant correlation with age (Pearson $r^{2}=0.16, p<0.002$ ). The small dependence of LCI on age is best summarised by a normal range ( $95 \%$ limits of normality) in adults of 5.9 to 7.5 and in children ( $\leqslant 16$ years) of 5.3 to 7.3. A weak relationship between height and LCI in the combined cohorts disappeared on multiple regression analysis. LCI was unrelated to gender of subject. By contrast, $\mathrm{FEV}_{1}$ varied between $76 \%$ and $133 \%$ predicted in the same group of 60 healthy adults and children.

\section{LCI in non-CF and CF adults}

The group mean (SD) LCI in adult healthy controls was 6.7 (0.4) (range 6.0-7.8) with 95\% limits of normality calculated as 5.9 to 7.5. In patients with CF the group mean (SD) LCI was 12.8 (3.6) (range 6.3-20.4), $p<0.001$ compared with healthy controls. The mean (SD) $\mathrm{FEV}_{1}$ was also significantly different between the two groups (102 (12)\% predicted in healthy controls vs 68 (23)\% predicted in patients with CF, p<0.001).

Figure 3 shows the relationship between $\mathrm{FEV}_{1} \%$ predicted and LCI for healthy controls and adults with CF. In controls LCI was restricted to a narrow range but, in patients with CF, LCI increased with reducing $\mathrm{FEV}_{1} \%$ predicted $\left(r^{2}=0.69\right.$, $\mathrm{p}<0.001)$.

There were 10 patients with CF with $\mathrm{FEV}_{1} \geqslant 80 \%$ predicted, all but one of whom had LCI above the upper limit of normal. By contrast, LCI was marginally raised in only two healthy adults (measuring 7.7 and 7.8). The sensitivity of LCI for detecting CF was $97 \%$ compared with $70 \%$ for $\mathrm{FEV}_{1}$.

\section{Repeatability of washout at same visit}

A washout test was excluded if the measured FRC differed by $>10 \%$ from both of the other two washouts. In adult subjects this resulted in the exclusion of a total of seven tests, representing $<3 \%$ of the total number of repeats from both healthy volunteers and patients with CF. Three tests were excluded from the paediatric cohort, representing $9 \%$ of the total number of washout repeats. All three washout repeats from an additional single adult healthy volunteer could not be analysed because they were unable to achieve a regular and reproducible breathing pattern. All three repeats from an additional healthy child (age 8) were also excluded because of evidence of an air leak.

After exclusion of these repeats, the mean (SD) intra-subject coefficient of variation (CV) for FRC derived from repeat washout manoeuvres on the same visit was 3.2 (1.9)\% for adult healthy volunteers, 3.9 (2.1)\% for healthy children and 3.5 (2.3)\% for patients with CF. The mean (SD) CV for LCI was 3.6 (2.1)\% for healthy adults, 5.4 (3.8)\% for healthy children and 4.4 $(2.8) \%$ for patients with CF. There was no significant correlation between the LCI CV and $\mathrm{FEV}_{1} \%$ predicted.

\section{Inter-visit reproducibility of $\mathbf{L C I}$ in healthy adults}

Repeat measurements of LCI were performed in triplicate on 16 healthy volunteers after a mean (SE) of 36 (10) days.

Table 2 Demographic data, spirometric parameters and lung clearance index (LCI) of healthy volunteers and patients with cystic fibrosis (CF)

\begin{tabular}{llll}
\hline & \multicolumn{2}{l}{ Healthy volunteers } & \\
\cline { 2 - 3 } & Children (age $\leqslant \mathbf{1 6}$ years) & Adults (age $\geqslant \mathbf{1 7}$ years) & CF patients \\
\hline $\mathrm{N}$ & 12 & 48 & 33 \\
$\mathrm{M} / \mathrm{F}$ & $7 / 5$ & $19 / 29$ & $21 / 12$ \\
Mean (range) age (years) & $11(6-16)$ & $33(19-58)$ & $30(17-49)$ \\
Mean (SD) FEV 1 (\% predicted) & $95(11)$ & $102(12)$ & $66(23)^{*}$ \\
LCl & $6.3(0.5)$ & & $13.1(3.8)^{*}$ \\
$\quad$ Mean (SD) & $5.6-7.1$ & $6.7(0.4)$ & $6.3-20.4$ \\
$\quad$ Range & $5.4(3.8)$ & $6.0-7.8$ & $4.5(2.7)$ \\
\hline
\end{tabular}

$\mathrm{FEV}_{1}$, forced expiratory volume in $1 \mathrm{~s}$; CV\%, coefficient of variation (\%) for intra-visit repeats.

${ }^{*} p<0.001$ vs adult healthy volunteers (Mann-Whitney $U$ test). 


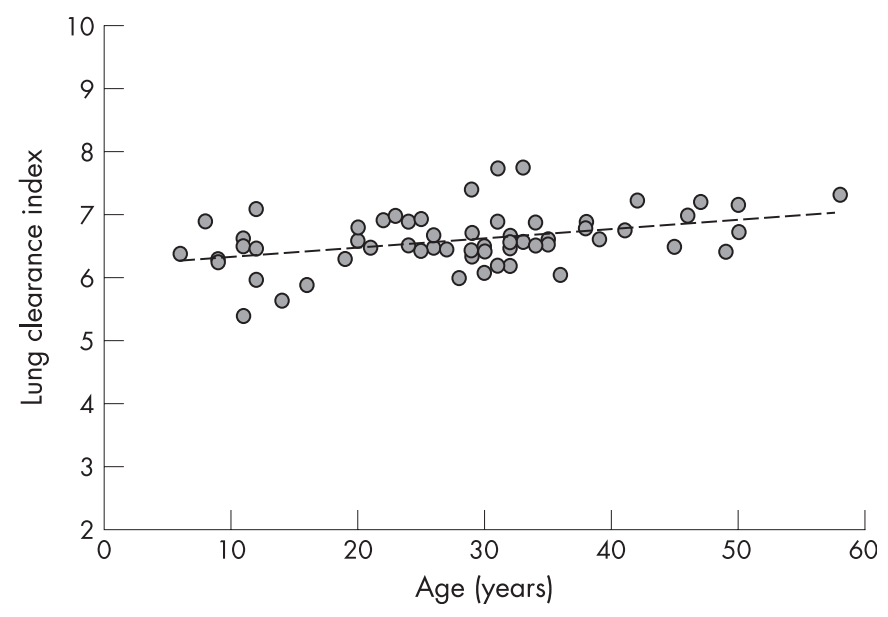

Figure 2 Effect of age on lung clearance index (LCI) in healthy volunteers. $\mathrm{LCl}$ remains within a narrow band of normal over an age range of 52 years. The broken line is the regression line, showing the extent of age-related increase in LCl.

A Bland-Altman $\operatorname{plot}^{20}$ of the difference between repeat measures and the mean of the measurements for LCI is shown in fig 4 . For FRC, the $95 \%$ limits of agreement between the two measurements were -0.43 to 0.45 litres and, for LCI, the $95 \%$ limits of agreement for the two measurements were -0.78 to 0.46. The inter-visit reproducibility of the FRC measurement was therefore approximately $400 \mathrm{ml}$ and that of the LCI measurement was 0.6.

\section{DISCUSSION}

This study has shown that the clinical measurement of inert gas washout is practical using equipment that is cheaper, more portable and has more sensitive gas signal resolution than the current MS standard. We have also shown for the first time that, in adults with CF, a simple measure of ventilation heterogeneity is more sensitive than $\mathrm{FEV}_{1}$ in detecting lung function abnormalities. Finally, we have shown that this measurement is reproducible both within and between visits, and that there is little change over a wide range of subject height and age.

In children with CF there is already increasing evidence that LCI is a more sensitive measure of early lung disease than $\mathrm{FEV}_{1}{ }^{9-1321}$ LCI has also been shown to correlate better with scores of airway damage on high-resolution CT scanning than spirometry. ${ }^{22}$ It may therefore fill an important gap in our ability to monitor airway function and disease progression noninvasively. Since only tidal breathing is required, it is particularly suitable for airways assessment in subjects who find complex respiratory manoeuvres difficult.

The potential of multiple breath washout measurements, however, has been hampered by the lack of a convenient method of performing them. ${ }^{12} 2123$ The original method for assessing lung clearance was the nitrogen washout. Although this avoids the need for a wash-in first, sufficient time must be allowed between tests for the end tidal nitrogen concentration to return to normal. ${ }^{18}$ The use of a MS to measure LCI by following changes in exogenous $\mathrm{SF}_{6}$ is now well described in children, ${ }^{12} 21$ and is probably the accepted gold standard technique in this population. The MS offers the additional advantage that it can measure a wider range of different gases, which is a useful option when measuring vital capacity single breath washouts. ${ }^{24}$ However, the MS is an expensive, temperamental and bulky piece of equipment that cannot readily be

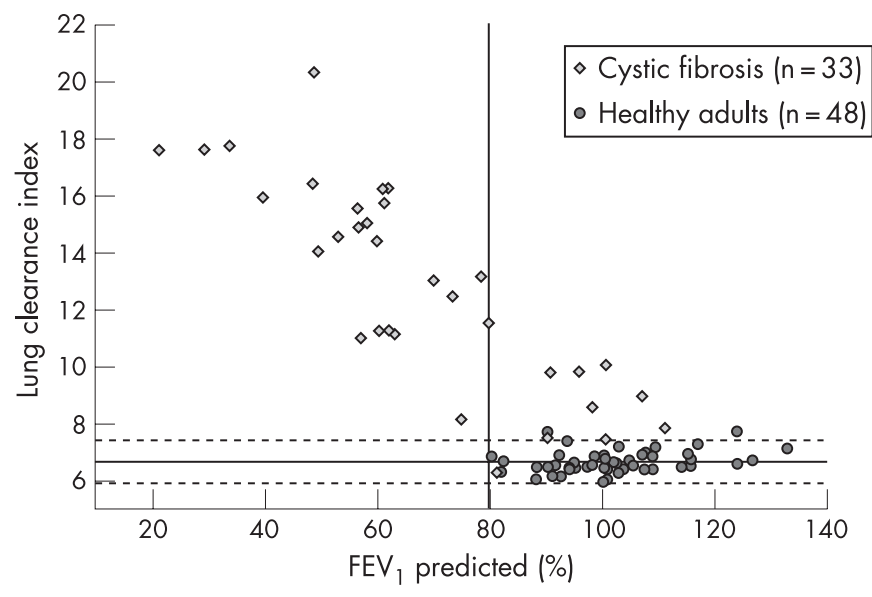

Figure 3 Lung clearance index ( $\mathrm{LCl}$ ) versus forced expiratory volume in $1 \mathrm{~s}\left(\mathrm{FEV}_{1}\right) \%$ predicted for adult healthy volunteers and patients with cystic fibrosis. The horizontal line represents the mean and the horizontal dotted lines the $95 \%$ limits of normality of the $\mathrm{LCl}$, calculated from the healthy adult population. The vertical line represents the lower limit of normal for $\%$ predicted $\mathrm{FEV}_{1}$.

taken out of the laboratory. In contrast, Innocor contains both the gas analyser and the pneumotachograph in a single unit that is both portable and robust. ${ }^{25}$ A supply of $\mathrm{SF}_{6}$ is required for both systems, but the concentration required for Innocor is 1/ 20 th of that used in the MS washouts, which reduces gas wastage and the potential environmental (greenhouse) effects of $\mathrm{SF}_{6}$.

The ideal comparison would be to compare the performance of both systems simultaneously, as has been done for other gas analysers. ${ }^{26}$ However, washouts would have to be performed at the operating range of the Innocor gas analyser since the response is not linear above $0.2 \% \mathrm{SF}_{6}$, but the signal resolution of the MS shows excessive noise at this level. Accepting this as a limitation of the current comparison, we have shown that the gas analyser is suitable for use in a multiple breath washout apparatus. The characteristics of the two analysers are summarised in table 2 of the online supplement (page 18). Our technical validation shows that the device with our modifications is capable of measuring gas volume by dilution with high accuracy. Despite operating at a much lower $\mathrm{SF}_{6}$ concentration, it produces washouts with a superior signal: noise ratio than a MS. Our comparison has also identified the possible limitations of the device imposed by the slower signal

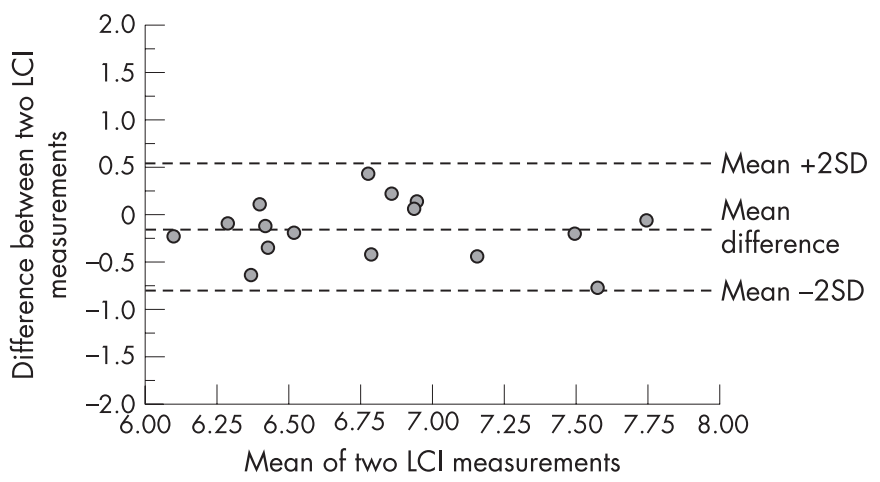

Figure 4 Bland-Altman plot of difference between lung clearance index (LCl) measured on two separate occasions (quoted as mean of triplicate repeats) and mean of the two measurements of $\mathrm{LCl}$. 
rise and fall time. The system is able to integrate flow and $\mathrm{SF}_{6}$ concentrations accurately at a physiological breathing rate of 10-30 breaths/min and should therefore be suitable for use in school-age children and adults. The response time may, however, limit the use of the method in neonates and preschool children with faster respiratory rates. ${ }^{21} 27$ Further in vitro assessment is required before using the analyser in this age group.

To date, we have used the modified Innocor to measure LCI in more than 100 patients and volunteers. From the data presented here, over $85 \%$ of subjects are able to complete all three washout manoeuvres without difficulty and generate reproducible measurements of FRC and LCI. Even for patients with CF, the whole process (wash-in and washout) usually takes little more than $10 \mathrm{~min}$, and considerably less in children. Despite the relatively uncontrolled conditions, the mean CV for repeat FRC is similar to that described in the literature, which varies from $3.5 \%$ to $6.7 \%$ for plethysmography and from $4.9 \%$ to $10.4 \%$ for helium dilution. ${ }^{28}$ The mean CV for LCI is also better than that described in children. ${ }^{11}$ Repeat measurements of LCI at a separate time point in a cohort of adult healthy volunteers also demonstrated good inter-visit reproducibility.

It has been shown that LCI may be influenced by large changes in tidal volume, respiratory rate or FRC. ${ }^{29}{ }^{30}$ We used tidal volume feedback to control tidal volume and respiratory rate within a range which should not affect the result. Since LCI is a ratio of cumulative expired volume and FRC, it is also independent of small changes in FRC over the physiological range. This is supported by the reproducibility of LCI and the narrow range of LCI in normal subjects found in the current study. Furthermore, because it is normalised for FRC, the normal range of LCI is largely unaffected by age, height or gender of subject. There was a weak but statistically significant rise in LCI with age. The clinical significance of this is unclear, since the magnitude of the difference (over a 52-year age range) remains very small and is less than inter-visit reproducibility. Serial dead space is known to affect LCI in infants and neonates. ${ }^{31}$ However, the change in normal values of LCI was in the opposite direction to that which would be caused by a greater dead space/tidal volume ratio (as found in infants). It is therefore possible that this represents a true effect of age on lung elasticity and hence gas mixing. By contrast, there is a wide range of "normal" for $\mathrm{FEV}_{1} \%$ predicted, which is influenced by the choice and accuracy of the normal range selected. ${ }^{32}$

The mean (SD) LCI determined here is very similar to that reported in the literature in children and adolescents. In preschool children (mean age 4 years) this has been reported as $6.9(0.4),{ }^{10}$ and in school-age children (mean age 11 years) as $6.5(0.5)^{11}$ and $6.3(0.4)^{9}$ in two different populations from the UK and Sweden, respectively. This supports our observation that LCI changes little with the age of the subject ( $>6$ years). This may be especially useful during long-term follow-up studies.

These are the first data on LCI in adults with CF; previous studies have only reported measurements in subjects up to 19 years of age. Even in adult patients with normal spirometry, the LCI may be markedly elevated, indicating significant "silent" lung damage. Some of the patients with normal FEV 1 had no symptoms and were on no treatment, the diagnosis of CF having been made incidentally. Yet, despite this, there was abnormal gas mixing in almost all cases. There is a risk that the extent of lung disease in such patients will be underestimated and hence undertreated.
While $\mathrm{FEV}_{1}$ is the currently accepted gold standard to monitor trials of new treatments for $\mathrm{CF}$, the rate of decline in this parameter has steadily reduced over the last decade. ${ }^{33}$ Power calculations show that many hundreds of patients would need to be treated over a year or more to see any beneficial effect of a novel therapeutic agent aimed at the basic defect. ${ }^{34}$ We have therefore instituted a large programme to assess novel biomarkers which could act as surrogates for $\mathrm{FEV}_{1}$. Ventilation heterogeneity is thought to be altered by small airways dysfunction, ${ }^{45}$ and measurements of this should therefore reflect the earliest pathology in CF-as has already been shown in children..$^{9-11}$ This is also the region of the lungs which is likely to be a key target for gene therapy. The choice of which subject to recruit into trials of gene therapy represents a conflict between those with sufficiently clear airways that the gene therapy complex can be delivered into the lungs and those with sufficient abnormality in lung function so that any improvement can be measured. LCI offers the ability to measure dysfunction in the airways of interest, and also to extend the range of patients suitable for these assessments.

We have shown that there is the possibility of a robust and compact apparatus to measure LCI that can be used in multicentre studies after relatively straightforward modification. This will permit us to assess LCI routinely in patients to obtain longitudinal data and, in particular, it may serve as a more sensitive measure of lung function after changes in therapy. The value of this technology may, however, extend beyond just CF and we anticipate that it may provide valuable information about the development and treatment of airways disease in other conditions. In particular, it may be useful in conditions that initially affect the small airways such as asthma, chronic obstructive pulmonary disease and obliterative bronchiolitis.

Acknowledgements: The authors thank all the volunteers who took part in this study. They thank Eddie Bergsten for his help in developing the software used to analyse the inert gas washout data and acknowledge the assistance provided by the Welcome Trust Clinical Research Facility at the Western General Hospital, Edinburgh.

Funding: This study was performed at the Welcome Trust Clinical Research Facility at the Western General Hospital, Edinburgh and at the Department of Child Life and Health, Royal Hospital for Sick Children, Edinburgh. Funding was provided by the CF Trust as part of the UK CF Gene Therapy Consortium.

Competing interests: None.

\section{REFERENCES}

1. Ramsey BW, Boat TF. Outcome measures for clinical trials in cystic fibrosis. Summary of a Cystic Fibrosis Foundation consensus conference. J Pediatr 1994; 124:177-92.

2. Macklem PT, Mead J. Resistance of central and peripheral airways measured by a retrograde catheter. J Appl Physiol 1967;22:395-401.

3. Brody AS, Klein JS, Molina PL, et al. High-resolution computed tomography in young patients with cystic fibrosis: distribution of abnormalities and correlation with pulmonary function tests. J Pediatr 2004;145:32-8

4. Cosio M, Ghezzo H, Hogg JC, et al. The relations between structural changes in small airways and pulmonary-function tests. N Engl J Med 1978;298:1277-81.

5. Verbanck S, Schuermans D, Meysman M, et al. Noninvasive assessment of airway alterations in smokers: the small airways revisited. Am J Respir Crit Care Med 2004;170:414-9.

6. Venegas JG, Winkler T, Musch G, et al. Self-organized patchiness in asthma as a prelude to catastrophic shifts. Nature 2005;434:777-82.

7. Samee $\mathbf{S}$, Altes T, Powers $\mathrm{P}$, et al. Imaging the lungs in asthmatic patients by using hyperpolarized helium-3 magnetic resonance: assessment of response to methacholine and exercise challenge. J Allergy Clin Immunol 2003;111:1205-11.

8. Venegas JG, Schroeder T, Harris S, et al. The distribution of ventilation during bronchoconstriction is patchy and bimodal: a PET imaging study. Respir Physiol Neurobiol 2005;148:57-64.

9. Gustafsson PM, Aurora P, Lindblad A. Evaluation of ventilation maldistribution as an early indicator of lung disease in children with cystic fibrosis. Eur Respir $J$ 2003;22:972-9. 
10. Aurora P, Bush A, Gustafsson P, et al. Multiple-breath washout as a marker of lung disease in preschool children with cystic fibrosis. Am J Respir Crit Care Med 2005;171:249-56.

11. Aurora $\mathbf{P}$, Gustafsson $\mathrm{P}$, Bush $\mathrm{A}$, et al. Multiple breath inert gas washout as a measure of ventilation distribution in children with cystic fibrosis. Thorax 2004;59:1068-73.

12. Aurora $\mathbf{P}$, Kozlowska W, Stocks J. Gas mixing efficiency from birth to adulthood measured by multiple-breath washout. Respir Physiol Neurobiol 2005;148:125-39.

13. Kraemer R, Blum A, Schibler A, et al. Ventilation inhomogeneities in relation to standard lung function in patients with cystic fibrosis. Am J Respir Crit Care Med 2005;171:371-8.

14. Becklake MR. A new index of the intrapulmonary mixture of inspired air. Thorax 1952; 7:111-6.

15. Miller MR, Hankinson J, Brusasco V, et al. Standardisation of spirometry. Eur Respir J 2005:26:319-38.

16. Quanjer PH, Tammeling GJ, Cotes JE, et al. Lung volumes and forced ventilatory flows. Report Working Party Standardization of Lung Function Tests, European Community for Steel and Coal. Official Statement of the European Respiratory Society. Eur Respir J Supp/ 1993;16:5-40.

17. Rosenthal M, Bain SH, Cramer D, et al. Lung function in white children aged 4 to 19 years: I-Spirometry. Thorax 1993;48:794-802.

18. Wanger J, Clausen JL, Coates A, et al. Standardisation of the measurement of lung volumes. Eur Respir J 2005;26:511-22.

19. Arieli R, Van Liew HD. Corrections for the response time and delay of mass spectrometers. J Appl Physiol 1981:51:1417-22.

20. Bland JM, Altman DG. Statistical methods for assessing agreement between two methods of clinical measurement. Lancet 1986;1:307-10.

21. Gustafsson PM. Inert gas washout in preschool children. Paediatr Respir Rev 2005;6:239-45.

22. Lindblad A, de Jong $P$, Brink $M$, et al. Measurements of ventilation inhomogeneity correlate better to structural changes on high resolution CT than conventional spirometry. Pediatr Pulmonol 2005;310-1.
23. Bush A, Davies J. Early detection of lung disease in preschool children with cystic fibrosis. Curr Opin Pulm Med 2005;11:534-8.

24. Gronkvist MJ, Emery MJ, Gustafsson PM. Mechanisms of ventilation inhomogeneity during vital capacity breaths standing and supine. Respir Physiol 2002; 129:345-55.

25. Ghofrani HA, Reichenberger F, Kohstall MG, et al. Sildenafil increased exercise capacity during hypoxia at low altitudes and at Mount Everest base camp: a randomized, double-blind, placebo-controlled crossover trial. Ann Intern Med 2004:141:169-77.

26. Fuchs SI, Buess C, Lum S, et al. Multiple breath washout with a sidestream ultrasonic flow sensor and mass spectrometry: a comparative study. Pediatr Pulmonol 2006;41:1218-25.

27. Beydon N, Davis SD, Lombardi E, et al. An official American Thoracic Society/ European Respiratory Society statement: pulmonary function testing in preschool children. Am J Respir Crit Care Med 2007:175:1304-45.

28. Hankinson JL, Stocks J, Peslin R. Reproducibility of lung volume measurements. Eur Respir J 1998;11:787-90.

29. Gronkvist M, Bergsten E, Gustafsson PM. Effects of body posture and tidal volume on inter- and intraregional ventilation distribution in healthy men. $J$ App/ Physiol 2002;92:634-42.

30. Bouhuys A, Lichtneckert S, Lundgren $\mathrm{C}$, et al. Voluntary changes in breathing pattern and N2 clearance from lungs. J Appl Physiol 1961;16:1039-42.

31. Schmalisch G, Proquitte $\mathrm{H}$, Roehr CC, et al. The effect of changing ventilator settings on indices of ventilation inhomogeneity in small ventilated lungs. BMC Pulm Med 2006;6:20.

32. Roca J, Burgos F, Sunyer J, et al. References values for forced spirometry. Group of the European Community Respiratory Health Survey. Eur Respir J 1998;11:1354-62.

33. Que C, Cullinan $\mathrm{P}$, Geddes D. Improving rate of decline of $\mathrm{FEV}_{1}$ in young adults with cystic fibrosis. Thorax 2006;61:155-7.

34. Davis PB, Byard PJ, Konstan MW. Identifying treatments that halt progression of pulmonary disease in cystic fibrosis. Pediatr Res 1997;41:161-5.

\section{Lung alert}

\section{Extracellular ATP in asthma airway inflammation}

A role for ATP as a pro-asthmatic mediator has previously been suggested from in vitro studies. This group investigated whether extracellular ATP and purinergic signalling are important mediators of airway inflammation in asthma, using experimental mice models and human subjects.

Rapid accumulation of ATP was observed in bronchoalveolar lavage (BAL) fluid after allergen challenge in subjects with allergic asthma and in experimentally sensitised mice. In the mouse model, ATP binding to purinergic receptors drove inflammatory chemotaxis and bronchospasm. Prevention of this increase in ATP using treatment with the ATP-hydrolysing enzyme apyrase to degrade ATP, or blockade of ATP effects using broad spectrum purinergic P2 receptor antagonists, down modulated all of the cardinal features of the asthmatic response. In addition, administration of exogenous ATP to mice enhanced Th2-type sensitisation to inhaled antigen, indicating that ATP may contribute to the development of asthmatic sensitisation by recruitment of Th2-inducing lung myeloid dendritic cells to mediastinal lymph nodes.

The authors conclude that ATP and purinergic signalling are important in the pathogenesis of asthma. Further studies are needed, but this may be an important therapeutic target for the future.

- Idzko M, Hammad H, van Nimwegen M, et al. Extracellular ATP triggers and maintains asthmatic airway inflammation by activating dendritic cells. Nat Med 2007:13:913-9

\section{Parekh}

Correspondence to: D Parekh, Medical SHO/ST2, Birmingham Heartlands Hospital, UK; dhruvey@hotmail.com

Thorax 2008;63:140 